\title{
Role of Telemedicine for Facio-Odontogenic Pain Management During COVID-19 Pandemic
}

\author{
Kritant Bhushan ${ }^{1} \cdot$ Prashant Kulkarni $^{2} \cdot$ Mansi Luthra Sharma ${ }^{3}$
}

Received: 26 July 2020/ Accepted: 16 September 2020/Published online: 25 September 2020

(c) The Association of Oral and Maxillofacial Surgeons of India 2020

\begin{abstract}
Introduction As the COVID-19 pandemic is increasingly griping the world, the entire landscape of existing patient management needs to be changed to avoid spread of Corona virus. In cases of emergencies, there is no option other than getting in-person consultations with doctors. But, in non-emergency patients, telemedicine can serve as a boon.

Material and methods In this article, we have tried to summarize the rules, regulations and protocols in accordance with government guidelines along with practical management for urgencies in dento-/oro-facial pains.

Conclusion If used wisely and as per recommendations, telemedicine is a very useful tool in the present COVID-19 pandemic, to avoid spread of infection to patients as well as health care workers.
\end{abstract}

Keywords Telemedicine $\cdot$ COVID-19 pandemic $\cdot$ Facial odontogenic pains

Kritant Bhushan

Kritant83@gmail.com

Prashant Kulkarni

drprashantak@gmail.com

Armed forces, Shimla, India

2 Nirmay Hospital, Pune, India

3 KD Dental college and hospital, Mathura, India

\section{Introduction}

Coronavirus disease 2019 (COVID-19) is an infectious disease caused by severe acute respiratory syndrome coronavirus 2 (SARS-CoV-2). It was first identified in December 2019 in Wuhan, Hubei, China, and has resulted in an ongoing pandemic. The first case of COVID-19 in India was reported on 30 January 2020. Ever since the emergence of these first cases, COVID-19 has become a public health crisis globally. To halt transmission, GOI impose the lockdown Pan India on 23 March 2020. COVID-19 spreads primarily when people are in close contact and one person inhales small droplets produced by an infected person [1,2]. The WHO recommends $6 \mathrm{ft}$ of social distance. As dentists are very prone and come under high-risk category in consideration of the risk, dental treatment may pose due to close contact with the patient oral cavity, saliva, blood and respiratory tract secretions; the Indian Government suspended regular dental treatments, allowing only emergency treatment [1].

In the current scenario of COVID-19 pandemic, telemedicine is emerging as a key technology for efficient communication and sustainable solution to provide essential health care services. Practice of dental surgery is an out-patient-based practice requiring a close contact with patient. Apart from that, pre- and post-treatment visits are required too. During these pre- and post-visits, patient needs to seat in waiting room, has to interact with receptionist and has to come in physical contact with other patient and their relatives [1]. In the time of corona pandemic, these visits can be avoided to some extent by telemedicine. 


\section{Guidelines for Use of Telemedicine}

Due to COVID-19 crisis, on 25 March 2020, Board of Governors (BoG) along with NITI Aayog recognized the need and released "Telemedicine Practice Guidelines" as an amendment in the Indian Medical Council (Professional Conduct, Etiquette and Ethics) Regulation, 2002 [1]. It is now legal to provide telemedicine consultation and prescription in accordance with the compliance of guidelines.

World Health Organization (WHO) has defined telemedicine as:

"the delivery of healthcare services, where distance is a critical factor, by all healthcare professionals using information and communication technologies for the exchange of valid information for diagnosis, treatment and prevention of disease and injuries, research and evaluation, and for the continuing education of healthcare providers, all in the interests of advancing the health of individuals and their communities".

Type of Telemedicine applications can be classified into four basic types [2-4]

1. Communication mode used: Audio (phone), video (Skype, WhatsApp, Google Duo), text based (WhatsApp messages, e-mail, mobile messages, etc.)

2. Timing of information communicated: real-time video/ audio/text interaction synchronous or asynchronous exchange of relevant information

3. First or follow-up consultation:

a. Emergency consult for immediate assistance or first aid, etc.

b. For non-emergency consult

1. First consult with any registered medical practitioners (RMP) for diagnosis/treatment/ health education/counselling.

2. Follow-up consult with the same RMP

4. According to the individuals involved:

a. Health professional to health professional, which gives easier access to specialty care, referral.

b. Health professional to patient which provides health care to the unreached population by giving them direct access to a medical professional.

\section{Seven Elements for Telemedicine Consultation} [5-8]

1. Telemedicine should be appropriate and sufficient as per context. Practitioners using telemedicine must maintain the same ethical and professional norms as of traditional face-to-face consult

2. Identification of doctor and patient. Telemedicine consultation should not be anonymous; both patient and the doctor need to know each other's identity

3. Mode of communication includes primarily three modes: video, audio or text, and all modes have some advantages over others but best mode is audio-visual mode which has certain limitation in remote area of country due to the lack of infrastructure

4. Consent: The consent can be implied if the patient initiates the telemedicine consultation. An explicit consent is needed if health worker, RMP or a caregiver initiates a telemedicine consultation

5. Type of consultation includes first consult and the follow-up consult

6. Patient evaluation includes all appropriate and adequate medical information about the patient's health must be obtained before making any professional decision

7. Patient management includes counselling to specific condition and prescribing medicines

\section{Medicines to Prescribe in Telemedicine}

List O: It will comprise those medicines which are safe and deemed necessary during public health emergencies. Antipyretics: Paracetamol, cough supplements: Lozenges, cough/common cold medications (such as combinations of Acetylcysteine, Ammonium Chloride, Guaifensen, Ambroxol, Bromhexene, Dextromethorphan), O.R.S. Packets, Syrup Zinc, supplements: iron and folic acid tablets, vitamin D, calcium supplements [7, 8].

List A: Medicines which are relatively safe with low potential for abuse and can be prescribed in first consultation and follow-up consultation like antifungal medications, antibiotics like penicillin G, ciprofloxacin gp, refill medications for chronic diseases such as diabetes, hypertension and asthma [7, 8].

List B: This list includes 'add-on' medications which are used to optimize an existing condition.

Prohibited list: It includes medicines that connect cannot be prescribed via tele-consultation. Medicines listed in Schedule X of Drug and Cosmetic Act, for instance, anticancer drugs, narcotics such as Morphine and Codeine $[7,8]$.

Format of prescription: Format is not mandatory; it is essential for the doctor to provide a signed scanned or digital copy of the prescription $[7,8]$. 
Records of patients: Proper records of the patient history, observations, findings and prescription to be maintained for minimum 3 years [7, 8].

Emergency situations. The doctor based on his/her professional discretion may (1) advise first aid, (2) counselling and (3) facilitate referral. In all cases of emergency, the patient must be advised for an in-person interaction with a doctor at the earliest.

\section{Telemedicine in Facio-Odontogenic Pain Management}

Practice of dental surgery is an out-patient-based practice requiring close contact with patient. In the time of coronavirus pandemic, the whole landscape of interpersonal interaction is changing, social distancing and personnel protection are becoming norms in everyday life, and dental practise cannot be any exception to that. Telemedicine needs to be introduced now in dental practise to avoid as much as possible non procedural interactions.

Advantages: Advantage of telemedicine in this scenario lies in pre- and post-operative visits. Before the actual procedure, frequently patient requires pre-medications to reduce the infection and relieve the pain, so that procedure can be carried out relatively painless. Apart from the above pre-medications, patients having co-morbid history require pre-procedure consulting to

1. show relevant report,

2. note down co-morbid history,

3. advise consultation with relevant medical team and fitness,

4. stop anticoagulants like aspirin,

During the course of time, like above mentioned, patients may require medication.

After the procedure has been done, patient may require consultation after that

1. to note the post-operative well-being

2. to clarify doubts

3. to contact in emergency manner

In otherwise healthy patients, these things can be carried out through telemedicine to a large extent.

\section{Application of Telemedicine in Facio-Odontogenic Pain Management}

As per guidelines issued by govt of India and DCI, procedures that can be carried out in COVID-19 pandemic can be divided into two categories:
1. Emergency procedures-procedures in which lifethreatening risk is involved, e.g. fast spreading infections, uncontrolled bleedings, trauma involving face

These cases need referral to emergency set-up or hospital set-up

2. Urgency procedures: Cases which require treatment on priority basis but are not life threatening, e.g. pulpitis, peri-coronitis, dental abcesses, unavoidable dental extractions, dento-alveolar trauma.

Conditions that are liable to consult via telemedicine are

- peri-coronitis

- pulpitis

- non-life-threatening acute and chronic space infections

- gum infections

- maxillary sinusitis

- neuralgic pain

- Bell's palsy

Peri-coronitis: It is judged by location and erupting status of tooth around third molar region. Age group of 16-35 years can give the clue. Treatment by telemedicine may include gargle $3 \% \mathrm{H}_{2} \mathrm{O}_{2}$ with 1:1 dilution with normal saline, proper oral hygiene, use of tablet Paracetamol $500 \mathrm{mg} 3$ times a day. In severe cases, Amoxicillin $500 \mathrm{mg}$ three times a day and Metronidazole $400 \mathrm{mg} 3$ times a day may be prescribed $[9,10]$.

Pulpitis: Pulpitis is associated with severe throbbing or sharp shooting pains with long history of associated carious tooth. They can be treated with common home remedy of clove oil applied to cotton pellet and inserted to carious tooth cavity. Additionally, tablet Paracetamol can be added as $500 \mathrm{mg} 3$ times a day. In severe cases, Amoxicillin $500 \mathrm{mg}$ three times a day and Metronidazole $400 \mathrm{mg} 3$ times a day may be prescribed [11].

Non-life-threatening acute and chronic space infections: These are associated with long-standing carious teeth, swelling in surround soft tissues. Pain will be severe in acute cases and moderate in chronic cases. Chronic cases are associated with pus draining fistulas and soar test in mouth. Non-life-threatening infections are generally not associated with fever and septicaemia. Treatment includes Co-amoxyclav 625 (Amoxicillin $500 \mathrm{mg}+$ Clavulanic acid $125 \mathrm{mg}$ ) thrice a day, Metronidazole $400 \mathrm{mg} 3$ times a day and Ibuprofen $400 \mathrm{mg} 3$ times a day [12-14].

Gum infections: These are relatively mild with associated minor abscesses some times. Associated pain will also be minor. It can be treated with good hygiene, tannic acid, astringent gum paint, gum massage. Abscess may need drainage by a qualified nearby doctor [15].

Mouth ulcers: Ulcers which are tender and less than 3 weeks of presence are generally aphthous ulcers. Such 
patients generally have recurrence history. Treatment for such patients includes balanced diet, multi-vitamins, local application gels containing local anaesthetic and choline salicylates $[15,16]$.

Oral Herpes infection: It is caused by herpes simplex virus and clinically manifested as tender blisters and rashes over lips and oral cavity, fever and lymphadenopathy. Disease is generally self-limiting and goes away in 1-2 weeks [15]. Treatment includes adequate hydration, balanced nutrition, oral antiseptic mouth washes and local application gels containing local anaesthetic and choline salicylates [16].

Maxillary sinusitis: It is clinically diagnosed by dull pain and heaviness lateral to nose and in cases with dental origin maxillary sinusitis, frequently pain in maxillary first molars. Management includes Tab. Paracetamol 500 mg 3 times a day and Cap. Amoxicillin $500 \mathrm{mg}$ three times a day for 5 days $[17,18]$.

Trigeminal Neuralgia: It is clinically manifested as sudden sharp shooting lancinating pain that is triggered after touching particular area distributed by one of the divisions of trigeminal nerve [19]. Cases that are newly diagnosed with trigeminal neuralgia will definitely need face-to-face consultation with expert since drugs used in management cannot be prescribed via telemedicine for first time. But, known cases of trigeminal neuralgia who are under treatment and whose proper medical history are known already and can be advised to manipulate the dosages as per patient needs, or even supplemental medications may be added.

Bell's Palsy: It appears as sudden expressionless face with deviation of corner of mouth to opposite side, drooling of saliva. In newly diagnosed cases, such patients can be advised medications like multi-vitamins, lubricant eyedrops and physiotherapy [20]. Prescription of antiviral drugs and steroids will require face-to-face consultation with expert. As in cases with trigeminal neuralgia, cases which are already under treatment and known for their medical history can be advised manipulation of medications as per need.

Limitation of telemedicine in Indian set-up: Poor telecom and cyber infrastructure and free distribution of medicines cannot be possible, and tele-consultation in patients with rural background may not be practical due to issues pertaining to education, network, etc.

\section{Conclusion}

In the time of COVID-19 pandemic, reducing the interperson interaction in Facio-Odontogenic Pain Management to a large extent can be done for in pre- and post-visits by telemedicine. During this pandemic, as a new edge tool, we all need to get used to with techniques as well as legal protocols associated with it, for the safety of ourselves, our staff, patients as well as relatives. Also, since long-term management of Facio-Odontogenic Pain is operative one, we need to consider limitations of the techniques limited to only medical management.

Funding None.

Compliance with Ethical Standards

Conflict of interest There are no conflicts of interest.

\section{References}

1. Telemedicine Practice Guidelines. https://www.mohfw.gov.in/ pdf/Telemedicine.pdf. Accessed Apr 26 Apr 2020

2. Enabling delivery of essential health services during the COVID 19 Outbreak: Guidance note. https://www.mohfw.gov.in/pdf/ Essential services during COVID19 updated 0411201.pdf. Accessed 13 May 2020

3. Chhabra HS, Sharma S, Verma S (2018) Smartphone app in selfmanagement of chronic low back pain: a randomized controlled trial. Eur Spine J 27:2862-2874

4. Mishra S, Kapoor L, Singh I (2009) Telemedicine in India: current scenario and the future. Telemed J e-Health 15:568-575

5. Chauhan V, Galwankar S, Arquilla B, Garg M, Somma SD, ElMenyar A et al (2020) Novel coronavirus (COVID-19): leveraging telemedicine to optimize care while minimizing exposures and viral transmission. J Emerg Trauma Shock 13:20-24

6. Cohen SP, Baber ZB, Buvanendran A, McLean LTCBC, Chen Y, Hooten WM et al (2020) Pain management best practices from multispecialty organizations during the COVID-19 pandemic and public health crises. Pain Med. https://doi.org/10.1093/pm/ pnaa12

7. WHO (1998) A health telematics policy in support of WHO's health-for all strategy for global health development: report of the WHO group consultation on health telematics, 11-16 December, Geneva, 1997. World Health Organization, Geneva

8. Telemedicine-opportunities and developments in member states (2010) 2nd edn. WHO press, Geneva. ISBN : 978-92-4-156414-4. https://www.who.int/goe/publications/goe_telemedicine_2010. pdf

9. Renton T, Wilson NH (2016) Problems with erupting wisdom teeth: signs, symptoms, and management. Br J Gen Pract 66:e606-e608

10. Cherry WR, Lee JY, Shugars DA, White RP Jr, Vann WF Jr (2012) Antibiotic use for treating dental infections in children: a survey of dentists' prescribing practices. J Am Dent Assoc 143:31-38

11. Eroglu CN, Keskin Tunc S, Erten R, Usumez A (2018) Clinical and histological evaluation of the efficacy of antimicrobial photodynamic therapy used in addition to antibiotic therapy in pericoronitis treatment. Photodiagn Photodyn Ther 21:416-420

12. Al Masan A, Dummer PMH, Farnell DJJ, Vianna ME (2018) Antibiotic prescribing for endodontic therapies: a comparative survey between general dental practitioners and final year Bachelor of Dental Surgery students in Cardiff, UK. Int Endod J 51(7):717-728 
13. Keswani ES, Venkateshwar G (2019) Odontogenic maxillofacial space infections: a 5-year retrospective review in Navi Mumbai. J Maxillofac Oral Surg 18(3):345-353

14. Dai T-G, Ran H-B, Qiu Y-X, Bo Xu, Liu Y-K (2019) Fatal complications in a patient with severe multi-space infections in the oral and maxillofacial head and neck regions: a case report. World J Clin Cases 7(23):4150-4156

15. Vincent SD, Lilly GE (1992) Clinical, historic, and therpeutic features of aphthous stomatitis. Literature review and open clinical trial employing steroids. Oral Surg Oral Med Oral Pathol 74:79-86

16. Collier PM, Neill SM, Copeman PW (1992) Topical 5-aminosalicylic acid: a treatment for aphthous ulcers. Br J Dermatol 126:185-188

17. Rosenfeld RM (2016) Clinical practice. Acute sinusitis in adults. N Engl J Med 375(10):962-970

18. Rosenfeld RM, Piccirillo JF, Chandrasekhar SS, Brook I, Ashok Kumar K, Kramper M, Orlandi RR, Palmer JN, Patel ZM, Peters
A, Walsh SA, Corrigan MD (2015) Clinical practice guideline (update): adult sinusitis. Otolaryngol Head Neck Surg 152(2 Suppl):S1-S39

19. Montano N, Papacci F, Cioni B, Di Bonaventura R, Meglio M (2012) Percutaneous balloon compression for the treatment of trigeminal neuralgia in patients with multiple sclerosis. Analysis of the potentially prognostic factors. Acta Neurochir (Wien) 154(5):779-783

20. Plumbaum K, Volk GF, Boeger D et al (2017) Inpatient treatment of patients with acute idiopathic peripheral facial palsy: a population-based health care research study. Clin Otolaryngol 42:1267-1274

Publisher's Note Springer Nature remains neutral with regard to jurisdictional claims in published maps and institutional affiliations. 